\title{
La cuestión de la subjetividad en un marco historico - cultural
}

\author{
Fernando L. González Rey \\ Universidad de Ia Habana
}

\begin{abstract}
Resumo
O artigo enfoca a questão da subjetividade da perspectiva da Psicologia Soviética e apresenta considerações sobre a epistemologia. O autor afirma que a epistemologia qualitativa toma possível a interdisciplinaridade. Palavras chaves: metodologia; Vygotsky; Rubinstein.
\end{abstract}

\section{The subjectivity question: one socio-cultural view}

\section{Summary}

The paper study the subjetivity question from the view of Sovietic Psychogology and presents considerations about the epistemology. The author says the qualitative epistemology take possible the interdisciplinarity.

Key words: methodology; Vygotsky; Rubinstein.

Una de las cuestiones presentes en la obra de L.S.Vygotsky, así como de S.L.Rubinstein, otro de los fundadores de la psicología marxista en la entonces Unión Soviética, fue el carácter holístico y complejo de la psique humana, ai que se refirieron desde la categoría personalidad, la cual fue considerada por ambos en su especificidad subjetiva, sin relaciones isomórficas con sus detenninantes. Ambos enfocaron la personalidad como sistema complejo, autorreguiado, que tenía una significación esencial en el sentido subjetivo de la experiencia humana. Aún cuando Vygotsky no llegó a desarrollar la categoría personalidad como uno de los pilares esenciales de su obra, como si lo hizo Rubinstein, su preocupación por la integración holística, compleja y dinámica de la subjetividad individual se expresa en múltiples momentos de su pensamiento, en lo que he llamado en trabajo anterior ${ }^{1}$ la representación general de Vygotsky sobre la psicología, la cual no tuvo tiempo de desarrollar orgánicamente en su teoría concreta.

En el estudio de los niños con deficiencias sensoriales, en un momento en que está muy influido por el pensamiento de Adler y Stem, influencia que refleja su sensibilidad por los procesos más complejos constitutivos de la personalidad, expresa (1995). : " El problema de la insuficiencia motriz es un bello ejemplo de aquella unidad en la heterogeneidad observada en el desarrollo del niño con defecto. La personalidad se desarrolla como un todo único, como un todo único reacciona ante la deficiencia, ante la alteración del equilibrio originada por ella y forma un nuevo sistema de adaptación y un nuevo equilíbrio en lugar del alterado. Pero precisamente debido a que la personalidad representa una unidad y actúa como un todo único, ésta, en el desarrollo, hace avanzar de un modo desproporcional unas u otras funciones diversas y relativamente independientes unas de las otras. Estos postulados, es decir, la variedad de funciones relativamente independientes en el desarrollo y la unidad de todo el proceso de desarrollo de la personalidad, además de no contradecirse unos alos otros, también como demostró Stern, se condicionan recíprocamente." (pag 14)

En la referencia anterior de Vygotsky, se puede apreciar su interés por ubicar la deficiencia dentro del contexto integral de la personalidad en desarrollo del deficiente, la cual se representa como proceso complejo que se desarrolla a través de los procesos simultáneos de su unidad y diversidad, idea que hasta hoy no se ha

\footnotetext{
${ }^{1}$ González Rey. F - L. S. Vygotsky; presencia y continuidad en el centenario de su nacimiento (inédito)
} 
impuesto dentro de las tradiciones intrapsíquicas que han dominado su estudio. Como se refleja en sus obras Pensamiento y Lenguaje, Psicología del Arte, así como en diversos miículos, Vygotsky siempre se representó los procesos psíquicos dentro de la identidad viva de la personalidad, aún cuando la propia complej idad de este planteamiento no tuvo tiempo de madurar en el desarrollo de su propia obra.

En el caso de S. L. Rubinstein, resulta curioso su interés por el tema de la subjetividad y la personalidad, el cual aparece en una obra de profundas implicaciones filosóficas y epistemo lógicas, que con frecuencia fue rechazada desde el trasfondo positivista que animaba las propias posiciones metodológicas de la psicología soviética. Rubinstein fue el primero dentro de la psicología soviética, en plantem'se la cuestión de la participación del sujeto como momento activo, inseparable del mundo objetivo en el proceso de construcción del conocimiento, principio que tuvo un fuerte desarrollo ulterior desde las posiciones del constructivismo. En este sentido escribió ya en 1922 : "Solo cuando de pmie del sujeto hay un acto de actividad creativa, su objeto es el mundo independiente, el ser objetivo"²

Al igual que Vygotsky, Rubinstein enfatiza la relación orgánica entre la personalidad y los procesos psíquicos, sobre la cual expresa (1967): "Todos los procesos psíquicos...ocurren en la personalidad y cada uno de ellos dependen de ella en su curso real" (pag 617), en otro momento de esta propia obra escribe: "Al estudiar los procesos psíquicos suele presentarse en el primer plano la ley determinante de como transcurre la percepción, el pensamiento, etc. Ahora bien, la percepción, el pensamiento del hombre, considerados como cierta vivencia concreta, como contenido de la vida de la persona, incluyen en sí, por lo común, no solo el reflejo de unos determinados fenómenos o de relaciones entre objetos, sino, además, el poner de manifiesto el sentido o significado que tales fenómenos y relaciones poseen para el hombre". (pag 172) En la referida cita vuelve a apreciarse como este autor en su concepción de reflejo, la que entiende más como presencia de la realidad en el conocimiento, que como reflejo de la realidad, se separa de la posición mecanicista de considerar el reflejo como una relación isomórfica entre la realidad y el conocimiento, definiendo con claridad el momento subjetivo de este proceso en el sentido que tiene para el sujeto.

La representación dialéctica, tanto de Rubinstein, como de Vygotsky en relación con la integración de la vida psíquica en una concepción holística y dinámica que ambos ubican en la personalidad, les conduce, por primera vez en la psicología, a romper con algunas de las dicotomías que siguen estando aún presentes en el desarrollo del pensamiento psicológico, como la dicotomía entre lo externo y lo interno, y de lo social y lo individual, lo cual solo puede lograrse con el desarrollo de una categoría más abarcadora y compleja, que permita integrar estos momentos como procesos vivos y contradictorios, definidos dentro de una misma naturaleza dei ser; la subjetividad. La subjetividad, sobre la que se expresa de forma explícita Rubinstein, más en un marco gnoseológico, aún cuando la identifica como estructura de sentido dei sujeto, y que tanto Rubinstein como Vygotstky la consideran de forma implícita en la constitución y el funcionamiento de la personalidad, les permite com prender al hombre como un sujeto en desarrollo comprometido con la cultura y con su medio social.

S. L. Rubinstein escribe (1967) : "La dimensión social no se mantiene como hecho externo con respecto al hombre: ella penetra y desde dentro determina su conciencia" (pag 19). Esta reflexión de Rubinstein expresa lo que será un atributo definitorio de la subjetividad; su carácter autorregulado y sistémico, dentro del cual se expresa solo aquella parte del mundo que tiene un sentido para el sujeto, que resulta relevante para él, no desde afuera, sino desde su propia constitución subjetiva, a partir de la cual se despliega todo su potencial contradictorio con el propio sistema dentro dei que resulta subjetivamente

\footnotetext{
${ }^{2}$ Tomando dei libra Concepciones filosófico - psicológicas de S. L. Rubinstein de los autores rusos K. A. Abuljanova y
} A. B. Bruchlinsky. 
constituida; la personalidad.

El concepto de personalidad se presenta como una instancia de carácter subjetivo, que no se diluye, ni se identifica linealmente con ninguno de sus determinantes, los cuales solo adquieren sentido desde la subjetividad que, desde esta comprensión, no define su carácter esencial por una constitución sustancializada estática, como ente cerrado en su propia naturaleza, sino como proceso en permanente desarrollo, comprometido con los distintos momentos de Ia vida dei sujeto individual en el que se constituye.

Este desarrollo inicial de la psicología soviética, donde el pensamiento marxista fue encontrando su potencialidad dialéctica en representaciones psicológicas, sobre todo en la presentación de la compleja relación dialéctica entre lo real y lo subjetivo, desafortunadamente no logró mantenerse en el curso de su propia historia, la que resultó intervenida desde las instancias políticas, factor que no puede determinarse en los rumbos posteriores de la psicología soviética, dentro de la cual la comprensión compleja y dinámica de los psíquico como forma particular de la constitución de lo real, dio paso a una concepción "objetivista" de la psique, que tuvo su mejor expresión en la teoria de la actividad de A.N.Leontiev, donde su carácter "objetal" resultaba dominante sobre su definición como forma de relación.

Con el desarrollo de la teoria de la actividad se identifican psique y actividad, perdiéndose la especificidad subjetiva de la constitución psíquica, lo cual conduce a la psicología soviética a un periodo donde lo esencial pasa a ser la demostración del carácter "objetivo" de los procesos psíquicos, identificándose esta objetividad en la relación directa entre estos y formas concretas de actividad, lo cual condujo a una vuelta de un experimentalismo positivista orientado al desentrafíamiento del vínculo directo entre la actividad y los diferentes procesos psíquicos. La actividad se convirtió en una supracategoría del pensamiento psicológico, definitoria de todos sus procesos.

En relación con la personalidad A.N. Leontiev escribió (1975): "Primeramente, es decir, hasta el esclarecimiento de los momentos esenciales que componen el proceso de actividad, el sujeto se mantiene fuera de los límites de la investigación. EI actúa solo en calidad de premisa de la actividad, de su condición. Solo el posterior análisis del movimiento de la actividad y de las formas de reflejo psíquico engendrado por él, conducen a la necesidad de incluir el concepto sobre el sujeto concreto, de la personalidad como momento interno de la actividad" (pag 159) . En esta cita del pensamiento de Leontiev se expresa con toda claridad la ubicación de la psicología dentro del marco de la actividad, considerada esencialmente como vía de desarrollo de diferentes formas de reflejo psíquico, en relación con la cual el sujeto y la personalidad solo representan momentos parciales, necesarios solo ante determinado nivel de desarrollo de la propia actividad.

La definición de la personalidad como momento interno de la actividad identifica a ambas por su estructura, con lo cual el lado activo del sujeto desatacado por S.L.Rubinstein desaparece, dando paso a una concepción totalmente objetivista de la psique, cuya fuente esta en la actividad externa, la que pasa a ser interna por el proceso de interiorización, que representa un tránsito lineal de lo externo a lo interno, momentos de la actividad que Leontiev considera idénticos por su estructura. Las críticas a esta posición, como he expresado en otros de mis trabajos ( 1982, 1985, 1986, 1989, 1993 y 1995), vinieron desde las más diversas posiciones dentro de la psicología soviética, incluso de autores formados dentro de esta dirección de pensamiento.

B.S.Bratus, uno de los seguidores jóvenes y discípulo de A.N.Leontiev escribio (1983): "La determinación del sentido solo como relación dei motivo con el objetivo en esencia cierra, limita este concepto a los marcos de una actividad tomada por separado. Entre tanto, las más importantes colisiones (al menos, de la personalidad adulta y madura) se desencadenan no en el plano "motivo - 
objetivo" ( como es descrito el sentido personal en términos de la actividad por A.N.Leontiev), sino en el plano "motivo - motivo", en las relaciones constituidas entre motivos" (pag 216) .

Con la hegemonía de la teoría de la actividad, se produjo un proceso creciente de "objetivación" y de reduccionismo sociologista dentro de la psicología soviética, el cual comenzó a ceder en la década del 70, con la emergencia de B.F.Lomov como una figura con poder político dentro de la psicología soviética, quien además fue uno de los pioneros en presental' la especificidad e importancia de la categoría comunicación en relación con la de actividad "objetal" propuesta por Leontiev, lo que desde mi punto de vista fue importante en el desarrollo de la psicología soviética. Unido a esto se desarrollo el simposium "El problema de la actividad en la psicología soviética" en 1977, donde se hizo una especie de balance crítico sobre el uso de la esta categoría, así como de sus limitaciones y potencial idades en aquel momento de desarrollo de la psicología soviética.

Dentro del referido simposium la investigadora N.I.Nepomnichaya, expresó (1977): "... la realización dei "enfoque de la actividad", que por si mismo tiene un significado decisivo para el desarrollo de la psicología materialista, ha dado lugar concretamente a una concepción unilateral y limitada sobre el objeto de la investigación psicológica. En el trabajo real tiene lugar una parcialización, una división de las distintas partes del objeto de estudio de la psicología. El pensamiento, los procesos sensoriales y la actividad son separados de la personalidad, y el concepto de personalidad se limita, por ejemplo, al de motivos y no incluye muchos otros aspectos dei sujeto integral" (pag 68).

En el mismo sentido se pronunciaron otros psicólogos soviéticos, incluso desde la propia década del 60, como V.N.Miasichev, uno de los fundadores de la Escuela de Leningrado, quien escribió (1960): "Sin embargo, ella (se refiere a la psicología soviética) todavía sufre de subdesarrollo, siendo su problema esencial que Ia psique es estudiada esencialmente por los procesos, pero su portador - la personalidad - se estudia insuficientemente. La actividad se investiga separada del actor. El objeto-los procesos de la actividad psíquica - se estudian sin el sujeto - la personalidad" (pag 6).

La ausencia del tema se la personalidad, tanto en la investigación básica como aplicada, fue muy significativa, lo cual, además de raíces teóricas, que tienen que ver con la propia interpretación del marxismo que se erigía dominante desde el poder político en aquel momento, también tuvo una profunda connotación ideológica, derivada del énfasis que se atribuía alos procesos macrosociales en la formación y desarrollo del hombre, el cual se consideraba como un reflejo de las condiciones externas, lo que llegó a implicar en término de las representaciones dominantes en la vida social en general la necesidad de trabajar esencialmente sobre las condiciones, quedando relegada la atención al desarrollo de las formas de relación entre las personas, lo cual marcó todas las esferas de la entonces psicología soviética.

La subjetividad como forma particular de los procesos humanos no interesaba, el desarrollo humano dependía de las condiciones en las cuales tenía lugar, por tanto, se trataba de garantizar la realización de las formas concretas de actividad, pasándose por alto la calidad de los vínculos desarrollados en las mismas. Las operaciones sustituyen al sujeto de la actividad, convirtiéndolo más en un sujeto reproductivo que creativo. K.A.Abuljanova escribe (1973): "Pese a las encarnizadas polémicas de los adictos a la explicación sociológica de lo psíquico y de los partidarios de la explicación fisiológica o cibernética, la posición de ambos grupos es idéntica en el orden metodológico. El afán de "cosificar", de materializar lo psíquico o asignarle el atributo de 
materialidad mediante su identificación con algo "distinto" conduce a un recurso elemental del pensamiento: el de reemplazar lo psíquico por algo "distinto"(pag 49).

\section{El desarrollo del tema de la subjetividad en la psicología}

El tema de la subjetividad no es nuevo en las ciencias sociales, sin embargo su desarrollo en la investigación y la producción teórica de las ciencias sociales patiiculares, exige un referente epistemológico totalmente diferente al de la perspectiva positivista que ha resultado dominante en estas. Dentro de la psicología, incluso las escuelas que se han desarrollado desde una tradición dinámica, que han enfatizado la naturaleza motivada del sujeto psicológico, el concepto de subjetividad no ha sido asumido de forma consecuente, entre otras cosas, porque la representación de ciencia dominante en ella no ha permitido trabajar con esta macrocategoría compleja.

Es interesante que la perspectiva que más se acerca al tema de la subjetividad en psicología, haya sido la que se desarrolló a partir del marxismo, cuyo núcleo dialéctico permitía la comprensión del objeto en términos complejos, a través de la pluridimensionalidad contradi ctoria de los elementos constituyentes del objeto de estudio. También fueron importantes antecedentes en el desarrollo del tema el psicoanálisis, la psicología humanista, y Ia obra de K. Lewin.

Sin embargo, la consideración del tema de la subjetividad dentro de las ciencias sociales no ha sido exclusiva de la psicología, expresándose en sociología, básicamente en la obra de Weber y sus continuadores, así como en el interaccionismo simbólico de Blumer. Weber ya había escrito (1964): " Oebe entenderse por sociología: una ciencia que pretende entender, interpretándola , Ia acción social, para de esa manera explicarIa causalmente en su desarrolIo y efectos. Por acción social debe entenderse una conducta humana (bien consista en un hacer externo o interno, ya en un omitir o permitir) siempre que el sujeto o las sujetos de la acción enlacen a ella un sentido subjetivo." (pag 5). Através del concepto de acción social Weber intenta integrar la social y lo individual, la cual aparece a vislumbrarse solo desde la comprensión del sentido subjetivo de esta integración.

En el mismo sentido que Weber enfatizando la integración de lo social y lo individual dentro de la constitución de la subjetividad humana, N. Elias expresa (1993): "En lugar de la imagen del ser humano como una "personalidad cenada" (...) aparece la imagen del ser humano como una "personalidad abiella" que, en sus relaciones con las otros seres humanos, posse un grado mayor o menor de autonomía relativa, pero nunca tiene una autonomía total y absoluta, y que, de hecho, desde el principio hasta el final de su vida, se remite y se orienta a otros seres humanos y depende de ellos"( pag 44). Elias retoma el término personalidad y lo ubica dentro del infinito proceso social donde ella existe con una autonomía relativa, proceso donde permanentemente se reconstituye y desarrolla como momento de Ia acción deI sujeto psicológico concreto en que aparece constituida.

Al reconocer la autonomía relativa de la personalidad, Elias entra sin proponérselo en la cuestión de su carácter histórico, pues la potencialidad de dicha autonomía es solo posible si la consideramos como sistema no dependi ente, en forma directa, de lo social. La personalidad se legitima en su propia historia, como sistema autoregulado que, constituyente del sujeto psicológico, resulta definitorio en la constitución o reconstitución del sentido subjetivo que ciertas formas de actividad y relaciones concretas tienen para él. La presencia de la personalidad dentro del sistema complejo de factores que participan en la definición del sentido subjetivo de la acción del sujeto, significa que esta no se diluye en el momento actual de la acción, sino que es constituyente del sentido subjetivo de la misma, condición que se define en su propia historicidad.

El sujeto, al entrar en cualquier nuevo espacio de expresión social, es portador de una condición 
subjetiva que se define por el desarrollo de su personalidad, por tanto, su acción dentro de este nuevo espacio no esta constituida solo por el sentido de las eventos sociales que aparecen en él, pues estos son inseparables de las estructuras de sentido de la personalidad.

El pensamiento de Weber, como de Elias y Blumer, aún cuando este último es criticado por su pragmatismo y la insuficiente atención que da alos procesos macrosociales en el desarrollo de su teoría, tienen, en mi opinión, una importante significación para el desanollo de la teoría de la personalidad que compatibilice su carácter histórico social y su naturalerza subjetiva. En nuestra opinión, el desarrollo de una teoría de la personalidad construida a partir de su constitución subjetiva, solo es posible desde una definición socio - histórica, que permita explicar Ia subjetivo en su especificidad, irreductible en términos de su constitución y desarrollo a ninguno de los sistemas que participan en su desarrollo.

Los autores que sefialamos, aún cuando dos de ellos provienen del campo de la sociología, Weber y Elias, mientras que Blumer es considerado tanto dentro de la sociología, como de la psicología social, tienen en común la consideración del sentido subjetivo que las acciones sociales tienen para los individuos como parte inseparable de su comprensión de los fenómenos sociales. En relación con esta Blumer expresa (1969): "el sentido determina la via en que el sujeto ve el objeto, la manera en la cual él esta preparado para actuar sobre él, y la vía en la cual él está preparado para hablar sobre él" .(pag II ).

Uno de los rasgos distintivos de la psicologia soviética, fue que se transformó en una psicología oficial, depositaria lineal de los rasgos distintivos del discurso político, desde el cual se enfatizó una concepción ahistórica de la producción intelectual, que llegó a legitimar su origen dentro del propio pensamiento soviético, rechazando como "burgués" formas anteriores de pensamiento, lo cual rompía con la necesaria continuidad e interrelación histórica de la producción científica. Solo esta situación permite explicar el desconocimiento de estos autores y la ausencia de un trabajo histórico serio, orientado apresentar en sus diferentes relaciones las formas históricas de producción del conocimiento psicológico.

La situación descrita se diferencia de los orígenes de aquella psicología, cuando la misma aún no había sido intervenida desde el nivel político y se inspiraba por la cosmovisión marxista de sus pioneros, quienes manifestaban un amplio dominio de las diferentes posiciones de la psicología de la época, al cual tuvieron acceso gracias a su posición abierta y no dogmática hacia todas las formas de producción de conocimiento, actitud realmente congruente con el pensamiento de Marx, el cual, como prácticamente todas las formas de pensamiento que han resultado dominantes en la historia de la humanidad, fue ideologizado a nombre de una política que muy poco tenía que ver con aquel a nivel filosófico.

En nuestra opinión la subjetividad representa ante todo una categoría ontológica, que expresa una forma de existencia del ser diferente de aquellas constituidas en el objeto de las ciencias no sociales, lo cual, como demuestran la mecánica cuántica y la biologia celular, no conduce a una ruptura epistemológica entre ambas, sino que exige reconocer sus especificidades a los efectos de su desarrollo.

Lo subjetivo no es aquello que no es, o lo que dificulta el conocimiento de lo que realmente es, sino que representa aquello que es de otra manera, que esta constituido por procesos y formas esencialmente diferentes a las de los "objetos sin subjetividad". La subjetividad designa una forma del ser constituida en niveles diferentes y que exige de una multiplicidad de formas de construcción teórica en las diferentes ciencias particulares, pues representa un aspecto esencial en la constitución de lo social, no solo de lo psíquico. Esta característica de la subjetividad como constitutiva del objeto de estudio de las ciencias sociales es uno de los aspectos que exige la interdisciplinaridad en su estudio.

Al plantearnos la subjetividad como definición ontológica la consideramos en su doble condición; de procesal y constitutiva, condiciones que se expresan simultáneamente a nivel social e individual, planos que 
se integran de forma contradictoria en ambos escenarios del desarrollo. Toda forma de subjetividad es social por naturaleza, la diferenciación entre la individual y la social está referida esencialmente a sus escenarios constitutivos, los cuales, aunque profundamente interrelacionados entre si, se manifiestan como sistemas diferentes, con historias propias.

La subjetividad humana como forma de lo real, existe en las estructuras simbólicas y de sentido subjetivo distintivas del hombre, las cuales no aparecen de una vez y por todas, como atributos universales del desarrollo, sino que se reconstituyen de forma diferente en el curso de este proceso a través de las formas de relación social en que el sujeto va desplegando su existencia social. El mundo donde el hombrevive se constituye subjetivamente en su personalidad, así como en las diferentes formas sociales en Ias que despliega su acción.

El marco de la subjetividad permite la integración de una serie de dimensiones de lo psíquico que históricamente han aparecido dicotomizadas en la historia del pensamiento psicológico, como son consciente - inconsciente, social - individual, interno - externo y cognitivo - afectivo, entre otras. Las mencionadas, aún cuando no agotan las dicotomías que históricamente se han engendrado con la tendencia a la fragmentación del conocimiento dominante en la epistemología positivista, serán objeto de atención en el presente artículo.

Con frecuencia, en l que siento muy influido por la tradición racionalista del pensamiento occidental, se enfatiza y hasta se identifica la dimensión subjetiva del hombre con lo simbólico, subordinando las emociones al funcionamiento simbólico, sobre todo a las estructuras y procesos de mediatización semióticas, como ha ocurrido en muchas de las lecturas del pensamiento de Vygotsky. En nuestra opinión las emociones constituyen procesos de significación, pero de una significación afectiva que, aunque aparezca constituida en estructuras simbólicas, dada la propia integridad de la personalidad humana, no depende de la mediatización simbólica para su definición en forma de sentido subjetivo.

Sartre expresa (1987): "Resulta, pues, imposible considerar la emoción como un desorden psicofisiológico. Tiene su esencia, sus estructuras particulares, sus leyes de aparición, su significación. No puede proceder desde fuera de la realidad humana. Es el hombre, por el contrario el que asume su emoción; por consiguiente la emoción es una forma organizada de la realidad humana.(pag 29). Sartre expresa en esta cita algo que ha estado ausente en la construcción psicológica; la naturaleza subjetiva de las emociones.

La emoción no es un producto secundario de otras formas o procesos constitutivos de la psique que puedan considerarse primarios o superiores en relación ella. Las estructuras de sentido de la subjetividad expresan una organización compleja, donde las emociones representan un aspecto constituyente más, no un subproducto de otras funciones. Como en toda organización compleja, la multidimensionalidad constitutiva de las estructuras subjetivas no esta subordinada a uno de los sistemas que la integran. El carácter motivado de las estructuras simbólicas no se subordina aios procesos de mediatización y producción definidos por ellas, sino que aparece como momento constitutivo de las mismas a través de procesos de autoregulación característicos del sistema dentro del que estas estructuras existen; la personalidad.

La subjetividad humana tiene como caracetrísticas generales las siguientes:

- Representa una organización constitutiva de cada momento de la acción del sujeto, la cual interviene activamente en la constitución de su sentido subjetivo, pero no como un determinante rígido y a priori, sino como parte del propio proceso constitutivo en que se define el sentido subjetivo de toda 
acción humana. La organización constitutiva de la subjetividad humana es la personalidad.

- La subjetividad tiene carácter histórico. Lo social deviene subjetivo en la propia historia individual, constituyéndose en otro sistema; la subjetividad. Esta se legitima a través de su propia historia y de las necesidades que en la misma se desarrollan. Las adquisiciones de cada nuevo momento del desarrollo, pasan a formar parte de las configuraciones que definirán nuevas adquisiciones en este proceso, las cuales representarán siempre un nuevo momento constitutivo en relación al anterior, nunca una extensión acumulativa de aquel.

- La subjetividad se expresa como sistema en constante desarrollo. El desarrollo es una cualidad del sistema que tiene lugar a través de la acción del sujeto psicológico, quien de forma permanente se compromete en nuevos vínculos sociales que, constituidos en y a través de su personalidad, representan, simultáneamente, un momento de cambio de esta en el curso mediato de su existencia.

- La subjetividad se expresa de forma diferenciada en un sujeto concreto, por tanto, su construcción teórica no puede ser supra individual. El sujeto en su dimensión reflexiva, vivencial e interactiva, es parte de su propio desarrolla, cuyas fuerzas no son externas a él, sino parte constitutiva de su momento actual.

\section{La categoria personalidad en una psicología histórico-cultural}

Durante mucho tiempo la categoría personalidad se identificó por atributos inherentes a ella, los que aparecían como elementos cristalizados a nivel individual, cuya relación con lo social se consideraba como una relación entre elementos esencialmente diferentes por naturaleza. Así, los diferentes sistemas teóricos que han resultado más relevantes en el estudio de la personalidad, siempre la han definido en torno a elementos invariables inherentes a ella, por ejemplo, en el caso de Freud, la personalidad aparecía como un sistema de instancias constituidas alrededor de la libido y del material reprimido, el que aparecía identificado y totalmente definido en el ello, la psicología humanista, aún cuando nos presenta una concepción más próxima a la subjetividad que la concepción biologicista y homeostática de Freud, considera sus principios dinámicos como inherentes a la naturaleza humana, los cuales aparecen a través de categorías diferentes, según el autor, en términos como la tendencia a la actualización, la realización o el sentido de la vida.

Definir la personalidad como expresión de la constitución subjetiva individual, implica el no reconocimiento de categorías constitutivas a priori, pasando a ser definitoria la propia constitución subjetiva de la experiencia del sujeto, la que toma formas diversas en sus diferentes actividades y. relaciones. En este sentido el planteamiento de una teoría de la personalidad desde el marco en que pretendemos definirla, implica la definición de unidades constitutivas capaces de articularse de forma flexible en los diferentes momentos de la experiencia del sujeto, definiendo su sentido subjetivo en el curso inepetible de la misma.

La diferenciación que supone el estudio de la personalidad desde su constitución subjetiva, nos lleva a buscar unidades psicológicas que no se definan por su contenido, sino desde por una forma particular de constitución capaz de dar cuenta de la configuración subjetiva diferenciada de eventos, hechos y personas constitutivas de la experiencia social del sujeto estudiado. En este sentido, por ejemplo, el concepto de Complejo de Edipo nunca podría representar un proceso general de la experiencia humana, sino una de las formas posibles de su constitución subjetiva a partir de la especificidad del vínculo entre el niño y sus padres, de la cualidad particular del mismo, 
y no una expresión de la naturaleza y las pulsiones de aquel.

Consideramos la personalidad como un sistema abierto de configuraciones subjetivas que expresan el sentido subjetivo de las diferentes formas de actividades, relaciones y de la experiencia integral del hombre, sin embargo, las unidades subjetivas en que se constituye la experiencia en la personalidad, se modifican y desarrollan en el curso de la propia experiencia. Las configuraciones se desarrollan a partir de la multiplicidad de emociones que aparecen en el curso de la acción humana, las cuales dan lugar a estados dinámicos que se integran entre si en la definición del sentido subjetivo de las mismas. Las configuraciones representan categorías complejas que se expresan a través de la integración de los elementos diversos que la constituyen durante el desarrollo humano.

Las configuraciones designan en términos de sentido subjetivo toda la experiencia humana, integran lo diverso de esta experiencia en estructuras de sentido que no guardan una relación directa y observable con ella. En este sentido es que afirmamos que las configuraciones se constituyen en el curso del desarrollo humano, eliminando términos como el de apropiación e interiorización, los cuales, por su propia definición, además de por su historia en el curso dei pensamiento psicológico, han enfatizado más la conversión de lo externo a lo interno, que la constitución de lo externo dentro de un sistema esencialmente diferente, que lo incorpora en sus propios términos; la personalidad.

En esta definición de las configuraciones como unidades constituidas, desaparece la falsa dicotomía de lo externo y lo interno, pues toda influencia externa es relevante al desarrollo humano solo cuando se integra a él en los términos en que el desarrollo tiene lugar, es decir, en estructuras de sentido, desde las cuales desplegarán su potencial contradictorio de forma mediata dentro del sistema de la personalidad. Por tanto, lo social como determinante del desarrollo humano aparece subjetivado dentro de la propia configuración de la personalidad, con lo cual deja de ser un determinante externo en relación a ella, para convertirse en parte de su propia constitución.

En esta lógica que presentamos, lo subjetivo tampoco es identificable con lo interno, pues se objetiviza en la acción del sujeto, a través de la cual pasa a formar parte de la propia realidad social, integrándose a otros aspectos de la misma que modifican el sentido original de su expresión por el sujeto, actuando desde otra dimensión de sentido en el curso de su experiencia social. Lo externo y lo interno se integran dentro de otra dimensión constitutiva de la realidad; la subjetividad, pasando a formar parte de otra naturaleza que los asimila en su carácter contradictorio, lo que resulta definitorio de su especificidad cualitativa.

Las configuraciones subjetivas de la personalidad representan complejas unidades de constitución subjetiva, las que representan un unidad funcional cognitivo - afectiva, integración que no viene de "afuera", definida por las características objetivas de una actividad o relación concretas, sino por el sentido que tiene el despliegue del sujeto dentro de las mismas para el desarrollo de la personalidad, sentido que solo se define por la inserción de la experiencia en la trama de sentidos que caracteriza el proceso de desarrollo en sus diversos momentos

Las configuraciones, como afirmamos más arriba, no representan unidades estáticas, portadoras de un mismo sentido subjetivo que se vuelve atemporal a partir de su aparición, sino formaciones históricas que van evolucionando en el propio curso de las actividades y relaciones que constituyen a nivel subjetivo. Portanto, su propio sentido subjetivo evoluciona constantemente en el marco de las formas de relación que expresan, así , por ejemplo, un padre puede tener un sentido en la vida de un niño, que cambie radicalmente con la entrada de aquel en la adolescencia, momento en que el sentido de las relaciones cambia, no solo por lo que acontece en el curso de la misma, sino por las propias 
modificaciones que se producen en los interlocutores ante las nuevas exigencias de aquella .

Las configuraciones no cambian solo en su constitución como formas particulares de la personalidad, sino que se integran y desintegran entre si ante los diferentes momentos de acción del sujeto, procesos en los que tienen un papel esencial sus emociones y reflexiones en el enfrentamiento con la situación, las que afectan de formas muy diversas, y ajenas a su intencionalidad, los procesos de autoregulación de la personalidad. Los momentos de reconfiguración de la personalidad implican la reorganización de configuraciones diferentes dentro de otras más abarcadoras, lo cual tiene lugar como proceso de autoregulación. Este proceso esta muy influi do por la acción del sujeto.

Así, por ejemplo, ante una situación comprometida con la profesión, un sujeto experimenta vivencias asociadas con la figura de su padre, cuyo sentido resulta esencial en la manera en que enfrenta la situación, lo cual pudo producirse de forma casual, por la presencia de algún elemento dentro de la situación interactiva, que le puso en contacto con otra esfera de sentido de la personalidad que se integró a la constitución subjetiva de la situación. Fenómenos de esta naturaleza se han hecho explícitos en nuestras investigaciones empíricas, unas veces a través de la construcción teórica indirecta de los mismos, y otras, por asociarse con la capacidad de representación del sujeto, que le permite su expresión intencional.

La personalidad no se agota en su organización configuracional, incluyendo en su desarrollo otros procesos y unidades subjetivas sobre los cuales nos resulta imposible detenernos en los límites del presente artículo, los cuales hemos presentados en diversas publicaciones anteriores (1985,1989,1995). Sin embargo, el concepto de personalidad que proponemos resultaria insuficiente sin el desarrollo de la categoria sujeto, la cual representa el carácter activo y diferenciado del individuo, una de cuyas consecuencias es el carácter abierto de la personalidad como sistema, que permite su reafirmación y compromiso social en cada uno de los momentos de la acción del sujeto.

El sentido subjetivo de los diferentes momentos de experiencia del sujeto concreto, no es algo que viene "de dentro", como se ha comprendido la determinación de la conducta desde las teorias intrapsíquicas de la personalidad, las cuales no lograron trascender los límites del determinismo mecanicista lineal en la comprensión de la relación personalidad conducta, la que se ha sido depositada fuera o dentro del sujeto, pero que nunca ha considerado a este como momento activo de constitución de esta relación.

Entendemos el sujeto como el individuo concreto, consciente, interactivo e intencional, que actúa dentro de su tiempo presente, independientemente del sentido subjetivo en que su noción de temporalidad aparezca constituida. El carácter interactivo del sujeto no se reduce a la interacción actual, definida por su presencia física en Ia misma, sino que la comprendemos como proceso dialógico que se expresa en su propia constitución subjetiva.

La constitución del sentido subjetivo de las diferentes formas de acción del sujeto tiene un carácter complejo, donde se integran los aspectos constitutivos de la personalidad, con las vivencias y reflexiones definidas en las situaciones que el sujeto enfrenta. Los aspectos constitutivos de la personalidad mediatizan las emociones que aparecen ante la nueva situación, representando un elemento de sentido en su definición, sin embargo, estas se organizan dentro de otras influencias dinámicas, definiéndose su sentido subjetivo en el conjunto de estos elementos, lo que hace imposible su explicación por una relación lineal con estructuras de la personalidad definidas previamente.

La personalidad no es una estructura anticipatoria que actúa a través de una relación de causalidad lineal con el comportamiento dei sujeto, la personalidad es un momento constitutivo del 
sentido subjetivo de las acciones actuales del sujeto, que se transforman en estructuras de su constitución subjetiva a través de la multiplicidad de emociones y sistemas de información involucrados en el curso de su expresión. Esta expresión tiene lugar a través de múltiples formas de comunicación, que tienen sentido no solo ante la mediatización de Ias formas constitutivas de la personalidad, sino por su propio lugar dentro de la organización de la subjetividad social.

Las decisiones del sujeto no son producto de fuerzas externas a él, sino resultante de un proceso activo, donde la decisión es construida dentro de un conjunto de elementos dinámicos diversos que, actuando dentro del proceso, no son necesariamente concientizados por el sujeto como momento de su decisión. La propia decisión, una vez asumida, puede convertirse en elemento constitutivo de diferentes configuraciones de la personalidad. La decisión, que en un momento dado expresó el carácter activo y reflexivo dei sujeto, una vez tomada se constituye a nivel subjetivo, pues se organiza dentro de las estructuras de sentido de la personalidad, proceso que ocurre no por la intención que la inspiró, sino por las diversas emociones producidas en el curso de la misma.

Unido a lo anterior, las consecuencias de la decisión se objetivizan en nuevas formas de lo social, las cuales se estrcuturan en la trama de lo real que el sujeto percibe como externa y que, sin embargo, se desarrolla en el curso de sus propias construcciones, las que son inseparables de la realidad percibida. este proceso expresa una objetivación de lo subjetivo.

La categoría sujeto, define la construcción teórica de la personalidad en un marco procesal y diferenciado que nunca a resultado dominante en la representación dominante en la psicología, lo cual aumenta considerablemente el valor heurístico de esta categoría y su significación para las diferentes esferas de desarrollo de la psicología.

La personalidad es el sistema constitutivo de la subjetividad individual, y a su vez es un momento constitutivo de la subjetividad social, dentro de la cual se desarrolla a través de la acción del sujeto en sus diferentes formas de inserción social. El sujeto representa un momento permanente de integración entre la subjetividad social y la individual.

Por otra parte la categoría sujeto deviene en momento esencial para la superación de las dicotomías consciente-inconsciente y cognitivo afectiva, a las cuales nos habíamos referido en momento anterior del presente artículo. La conciencia no representa ni un sector constitutivo de la vida psíquica, como en determinado momento se le consideró en el psicoanálisis, así como tampoco las relaciones entre cierto tipo de funciones psicológicas, como la definió el propio Vygotsky en un momento de su obra. La conciencia se define en la acción intencional del sujeto, Ia que se realiza a través de su lenguaje y reflexividad, momentos esenciales de la acción consciente. Por otra parte, esta acción consciente siempre se desarrolla dentro de relaciones concretas de comunicación, el sujeto estructura su expresión consciente en su intención de comunicación con los otros.

La intencionalidad del sujeto es un momento esencial del desarrollo de la conciencia. El proceso de pensamiento es uo ejercicio permanente de conciencia, donde simultáneamente el sujeto avanza en la construcción de un proyecto que anticipa, retroalimentándose sobre él a través de su confrontación con los otros, dentro de la cual queda constituido en estructuras que resultan comunicables que se desarrollan en el propio proceso de comunicación. La actividad consciente del sujeto se desarrolla por procesos de la cognición organizados en Ias estructuras simbólicas del lenguaje, pero esta no se orienta hacia una realidad definida en abstracto, sino a una realidad constitui da por su sentido subjetivo para el sujeto, por tanto, la cognición, así como la intencionalidad del sujeto, responden también a las necesidades resultantes del sentido que tiene para élla realidad que enfrenta. 
La actividad cognitiva del sujeto, por tanto, es simultáneamente motivada, generando en su propio curso un conjunto de emociones que actúan como autoreguladoras de la misma, integrando el proceso de producción cognitiva en el sistema complejo de sentido definido por la personalidad. La personalidad como nivel de construcción teórica de lo psíquico no representa una supracategoría sustitutiva de los otros procesos de la vida psíquica, sino un nivel más integral y complejo en el abordaje de los mismos. Como macrocategoría la personalidad permite estudiar en otra arista de su constitución subjetiva, procesos y funciones tradicionalmente trabajados en relación con otros de sus aspectos constitutivos, como el pensamiento, la creatividad, el aprendizaje, etc.

La personalidad no es un sistema cerrado, proveedor de respuestas directas ante problemas concretos, ella es ante todo un escenario para construir lo estudiado en la multiplicidad de procesos que lo definen cualitativamente, los cuales son articulables dentro del sistema representado por ella. La personalidad es un escenario para la construcción teórica, para la explicación de funciones psíquicas diferentes en diferentes niveles y formas de su constitución subjetiva.

La personalidad, más que una categoría de exclusión, como muchas veces ha aparecido en la historia del pensamiento psicológico, donde se ha pretendido definir a través de un conjunto de contenidos estáticos y universales que la constituyen, representa una categoría de inclusión, en el sentido que permite la integración de las diferentes funciones y procesos psíquicos en otro nivel de constitución subjetiva de los mismos; nivel más complejo que permite avanzar en su construcción pluridimensional y contradictoria en los marcos del sujeto diferenciado de los mismos.

Desde la perspectiva teórica que defendemos, la personalidad es inseparable del sujeto diferenciado en que se constituye, cuyas elaboraciones y construcciones tendrán un importante papel en la definición de indicadores para su construcción teórica. Esto, unido al tipo de constitución compIeja y diferenciada en que la hemos definido, hace de la personalidad un momento de desafío epistemológico y metodológico para la psicología.

\section{Desafíos epistemológicos deI estudio de Ia personalidad en psicología}

La presentación teórica que hemos presentado en el presente artículo es expresión de más de veinte afios de trabajo en el tema, y como toda construcción no expresa de forma directa la cantidad de momentos que hemos recorrido para llegar al punto de nuestra definición teórica actual. El avance de nuestro pensamiento hacia el momento actual en que nos encontramos en la construcción teórica del tema, ha transcurrido en medio de múltiples aproximaciones, en cuya producción se han seguido un conjunto de líneas dentro del momento empírico, las que nos han colocado frente al desafío de ir desarrollando posiciones metodológicas que no frenaran el curso del proceso de construcción teórica.

El desarrollo del tema de la personalidad, por tanto, no solo ha contribuido a un desarrollo teórico alternativo, sino que nos fue llevando por un proceso de reftexión metodológica que nos condujo de forma inevitable ai campo de la epistemología.

Como hemos expresado en otras publicaciones (1995, 1996), en los inicios de nuestro trabajo de doctorado en ellaboratorio dirigido por L.I.Bozhovich en 1976, quien fuera la única discípula de Vygotsky que intento desarrollar los principios explícitos de su obra en el estudio de la personalidad, asumimos Ia utilización de instrumentos cualitativos para el desarrollo de nuestra investigación, lo cual había sido una tradición de trabajo dentro dellaboratorio, sin embargo, esta tradición no se había reftejado en una reflexión organizada de carácter metodológico y muchos 
menos epsitemológico, sino que se desarrollaba de forma espontanea ante las propias exigencias del objeto de estudio.

Uno de los puntos débiles de la psicología soviética fue, como sefialamos más arriba, que en la búsqueda de materialización de lo estudiado, retorno a una perspectiva experimental atomizadora que, en lo metodológico la mantuvo dentro de los marcos de la epistemología positivista. La obra de los clásicos de dicho pensamiento, quienes desarrollaron un marco teórico que, sin dudas marco un hito en el desarrollo de la psicología, no se acompafió de una construcción metodológica alternativa, mientras que las interesantes reflexiones epistemo lógicas de Rubinstein tampoco tuvieron una expresión metodológica.

Analizando la historia de la psicología soviética es notaria la ausencia literatura metodológica y epsitemológica en el campo de la psicología y de las ciencias sociales en general, quizás por el riesgo que ello implicaba aios efectos de la objetividad de lo estudiado, la cual llegó a ser definitoria del carácter marxista de la investigación. El mayor compromiso de los temas epistemológicos con la creatividad filosófica, la cual estaba totalmente identificada con un discurso mimético que no evolucionaba en el tiempo, quizás fue uno de los elementos que dificultó el desarrollo de estos tópicos, sobre los cuales los clásicos de esta psicología tenían conciencia, lo cual se refleja tanto en la obra de Vygotsky, como en la de Rubinstein.

L.S.Vygotsky escribió: "...hay que encontrar una teoría que ayude a conocer la psiquis y no la solución del problema de la psiquis, no las fórmulas que resumen y sumen el resultado de la verdad científica (...) no se puede buscar en los maestros del marxismo la solución del problema, incluso ni siquiera una hipótesis de trabajo (porque ellas se crean en el terreno de la ciencia dada), sino el método de su construcción. Yo no quiero saber gratuitamente, habiendo recordado un par de citas, que es la psiquis; quiero aprender en todo el método de Marx,como construir la ciencia, como enfocar la investigación de la psiquis"3

La referida cita demuestra que Vygotsky consideraba entre los aspectos esenciales del marxismo para la psicología, su potencial epistemológico, comprendiendo el desarrollo teórico de la psicología como una responsabilidad de los psicólogos, con lo cual respondía a aquellos que pretendían el desarrollo de una psicología marxista a partir de la importación directa de categorías desarrolladas en el campo filosófico. Esta preocupación, sin embargo, no encontró lugar en el desarrollo posterior de la psicología soviética.

En el desarrollo del momento empírico de nuestras líneas de investigación, nos fuimos dando cuenta de la necesidad impostergable de desarrollar una posición epistemológica, que nos llevara a una mayor elaboración del marco metodológico en que nos movíamos, con lo cual comenzamos un trabajo en esta dirección, que se ha materializado en dos de nuestros últimos libros $(1993,1996)$, sin el cual hubiera sido imposible continuar avanzando en el desarrollo de una teoría alternativa de la personalidad, capaz de entrar como momento del desarrollo de diferentes líneas de producción teórica en la psicología.

La ausencia del tema de la epistemología en la psicología, determinó que cualquier intento alternativo al positivismo se identificara a sí mismo dentro de la hermeneútica o la fenomenología, las cuales han tenido un importante papel en formas alternativas de producción del conocimiento psicológico, a pesar de que muchos de los avances en la acción metodológica en psicología, fueron ante todo el resultado de las dificultades de los investigadores para desarrollar sus representaciones

\footnotetext{
${ }^{3}$ Tomada Ia cita deI libra "La psicologia soviética tal comoyo Ia veo"de laautoraM. Schuare (pag. 491,1990.)
} 
teóricas dentro de los límites rígidos impuestos por la metodología positivista, como por ejemplo ocurrió con el propio Freud.

La hermeneútica, así como la propia fenomenología, han representado tendencias generales del pensamiento filosófico que, aunque son un referente obligado de cualquier alternativa a la epistemología positivista, están orientadas más aios procesos de interpretación, que a la definición de alternativas metodológicas más completas en el campo de las ciencias particulares. En nuestra opinión, muy influi da por el pensamiento de Bachelard, las ciencias particulares deben avanzar en el desanollo de sus propias epistemologias, las cuales, aún cuando no sean incompatibles con el desarrollo de epistemologías generales a nivel filosófico, sean capaces de integrar la especificidad de sus respectivos objetos.

Entre las preocupaciones que nos llevó a una reflexión epsitemológica, estaba el creciente incremento de la investigación cualitativa en la psicología, la cual era para muchos incompatible con la utilización de instrumentos cualitativos (Kincheloe. J, 1991), mientras que para otros representaba más bien una forma de recolección de datos dentro de los mismos principios epistemológicos positivistas (Carlson. R, 1994). En nuestro criterio los instrumentos cualitativos y cuantitativos son compatibles a nivel metodológico, radicando la contradicción a nivel epistemológico, entre una epistemología que pretende llegar al conocimiento a través de procesos de significación estadística y conclusiones numéricas, y una epistemología que enfatiza la producción de conocimientos como un proceso cualitativo constructivo.

Hemos desarrollado nuestra posición epistemológica precisamente desde la definición cualitativa del proceso de construcción del conocimiento, lo cual nos ha conducido a identificar nuestro planteamiento como epistemología cualitativa, conscientes del potencial contradictorio de esta definición, el cual esperamos despejar en alguna medida en la explicitación de sus principios generales. El planteamiento epistemológico que defendemos responde a la propia naturaleza holística y procesal que atribuimos al objeto escogido para la producción de conocimientos; la personalidad y las formas constitutivas de la subjetividad social.

Coincidimos con Fenaroti cuando afirmó ( 1990): "Al hacer coincidir el conocimiento con la medición y con la medición exacta, en sentido cuantitativo, la ciencia moderna galileana ha realizado una operación reduccionista, esto es, ha reducido la naturaleza humana, compleja y no exactamente detallada, a la "unidimensionalidad" de los "meros hombres de hecho" (pag 86-87) A pesar cuando las propias ciencias que utilizan la modelación matemática, han sufrido una profunda transformación epistemológica, como es el caso de la física a través de la teoría de la relatividad y d la mecánica cuántica, donde las matemáticas aparecen como vías y momentos en la producción de conceptos y representaciones que no tienen una naturaleza cuantitativa, lo ciello es que este uso de las matemáticas no caracteriza a las ciencias sociales, cuya producción ha sido esencialmente cuantitativa, tanto en la investigación como en los procesos de diagnóstico, donde la naturaleza del objeto se ha expresado en término de cantidad, omitiendo toda construcción compleja sobre la misma.

Es en el contexto senalado en que se desenvuelven las ciencias sociales, donde he creído pertinente definir una forma particular de producción de conocimientos como epistemología cualitativa. La epistemología cualitativa se define, entre otros, por los siguientes principios esenciales:

- Considerar el conocimiento como un proceso constructivo - interpretativo. En esta 
definición el conocimiento no se define por sucesión de actos, ni por acumulación de resultados, sino como proceso que progresivamente va produciendo nuevas ideas y explicaciones no explícitas de forma directa en ninguno de los instrumentos utilizados para la producción del conocimiento. El dato nunca es asumido en calidad de resultado definitorio, susceptible de integrarse como definición parcial del objeto en correlaciones estadísticas capaces, mediante factores en presentar como diferente un nivel de resultados idénticos por su naturaleza con las unidades más simples que le sirvieron de base.

- Considerar el proceso de producción de conocimientos como proceso interactivo. El hombre se conoce a través de sus construcciones y expresiones diversas, la mayoría de las cuales tienen lugar en el proceso de comunicación, por lo que las ciencias humanas están obligadas a considerar la producción de conocimientos sobre su objeto a través de la comunicación con él, lo que coloca en una posición participativa tanto al investigador como al sujeto investigado.

Desde esta consideración epistemológica, la comunicación se convielle en el proceso subyacente sobre el que se desarrollan los diferentes momentos de cualquier metodología, sea orientada a un sujeto individual o social. El proceso de producción de conocimiento avanza a través de la comunicación entre los implicados, la que es definitoria en su implicación motivacional con la investigación, condición de la calidad y complejidad de su expresión, lo que garantiza la riqueza de indicadores a utilizar en la interpretación.

- La epistemología cualitativa legitima el nivel de lo singular en la producción del conocimiento científico. Los indicadores del momento empírico no adquieren su significación solo a través de la frecuencia de su expresión, sino del sentido que tienen dentro de cada momento de construcción teórica, por tanto su significación va a ser cualitativa, lo que es congruente con la definición epistemológica asumida.

Partiendo de lo anterior, el proceso de generalización es entendido como una construcción del nivel teórico, donde la generalidad de toda construcción se define por su capacidad explicativa sobre un conjunto de problemas diversos, lo que, a su vez, en ningún momento del proceso pierden su identidad diferenciada sobre la base de las categorías utilizadas para lograr su inteligibilidad teórica.

La epistemología cualitativa no niega el uso de los métodos cuantitativos, ni de los momentos descriptivos en la producción del conocimiento, los cuales son con frecuencia parte esencial del propio proceso de construcción del conocimiento, sin embargo, no considera estos como definiciones cuantitativas finales, sino como indicadores y momentos dentro del proceso, cuyo sentido se definirá progresivamente en términos explicativos.

Estas consideraciones epistemológicas están en la base del desarrollo de una metodología de investigación diferente, precisamente a dar cuenta de la representación compleja de la subjetividad, no solo a nivel de la personalidad humana, sino en otros niveles de su constitución. La construcción del conocimiento en ciencias sociales no puede prescindir de abordar su objeto en la pluralidad de determinantes en que se expresa.

Desde esta consideración epistemológica, la ciencia psicológica se desarrolla a través de todas las vías de ejercicio profesional del psicólogo, quien integra los indicadores diversos producidos en el curso de las mismas en la continuidad de su producción intelectual. La epistemología cualitativa permite romper los estancos intradisciplinarios rígidos que han dominado el 
escenario de la psicología, permitiendo tanto comprender la subjetividad en su naturaleza históricocultural, como el lugar de la misma, en su condición de macrocategoría del conocimiento psicológico, dentro de las diferentes esferas de la psicología.

\section{Referências}

Abuljanova, K. A. (1973). El sujeto de la actividad psiquica. Editorial Nauka. Moscú.

Abuljanova, K. A. (1980). La actividady la psicologia de la personalidad. Editorial Nauka. Moscú.

Abuljanova, K. A. (1989). La concepción filosófico-psicológica de S.L.Rubinstein. Nauka. Moscú.

Allport, G. (1967). La personalidad. Su configuración y desarrollo. Edición Revolucionaria.La Habana.

Blumer, H. (1969). Symbolic interactionism. Perspective and Method. Englewood Cliff, Prentice Hall.

Bratus, B. S. (1981). Hacia el estudio de la esfera de sentido de la personalidad. Vestnik de la Universidad de Moscú.Serie 14, Psicología, No 2.

Durkheim, E. (1972). Las reglas del método sociológico. Schapire. Buenos Aires.

Elias, N. (1993). El proceso de la civilización. Investigaciones sociogenéticas y psicogenéticas. FCE, Madrid.

Ferraroti, F. (1990). Una fe sin dogmas. Ediciones Península. Madrid.

González, F. (1983). La comunicación y su significado en la elaboración del problema de la personalidad. Psyjologichevsky journal.TA, No 4, Moscú.

González, F. (1985). Psicologia de la personalidad. Editorial Pueblo y Educación. La Habana.

González, F. (1989). La personalidad; su educación y desarrollo. Editorial Pueblo y Educación. La Habana.

González, F. (1993). Problemas epistemológicos de la psicologia. Editorial Colegio de Ciencias y Humanidades. UNAM. México.

González, F. Epsitemologia cualittiva y subjetividad. (libro inédito).

Lomov, B. F. (1981). Hacia el problema de la actividad en psicología. Psyjologichevskie journal. T.2, No 5.

Miasichev, V. N. (1960). Personalidady neurosis. Editora de la Universidad de Leningrado.

Nepomnichaya, N. I. (1977). Actividad, conciencia, personalidad y el objeto de la psicologia. Em: El problema de la actividad en la psicología soviética. Editorial Pedagoguica. Moscú.

Rubinstein, S. L. (1967). Principios de Psicología General. Edición Revolucionaria. La Habana.

Rubinstein, S. L. (1962). EI Ser y Ia Conciencia. Edición Revolucionaria. La Habana.

Shuare, M. (1990). La psicología soviética tal como yó Ia veo. Editorial Progreso.Moscú.

Vygotsky, L. S. (1967). Pensamiento y Lenguaje. Edición Revolucionaria.La Habana. 
Vygotsky, L. S. (1995). Tratado de Defectología. Obras Completas. Tomo V. Editorial Pueblo y Educación. La Habana.

Recebido em setembro 1998.

Encaminhado para revisores em outubro 1998.

Aprovado em outubro 1998. 